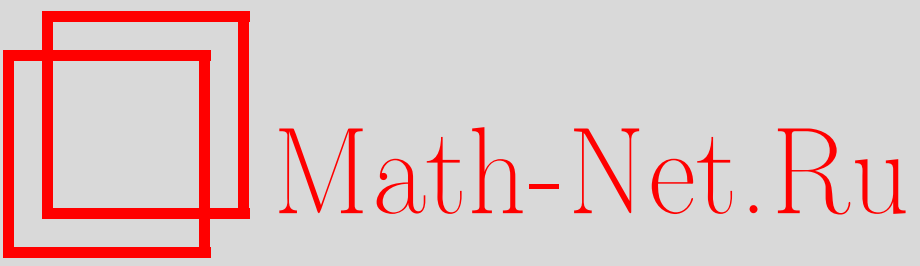

А. А. Жукова, Модель поведения производителя при наличии случайных моментов получения кредита и инвестиций, Матем. моделирование, 2020, том 32, номер 4, 16-30

DOI: https://doi.org/10.20948/mm-2020-04-02

Использование Общероссийского математического портала Math-Net.Ru подразумевает, что вы прочитали и согласны с пользовательским соглашением

http://www.mathnet.ru/rus/agreement

Параметры загрузки:

IP: 54.80 .73 .141

26 апреля 2023 г., 07:26:09 


\title{
МОДЕЛЬ ПОВЕДЕНИЯ ПРОИЗВОДИТЕЛЯ ПРИ НАЛИЧИИ СЛУЧАЙНЫХ МОМЕНТОВ ПОЛУЧЕНИЯ КРЕДИТА И ИНВЕСТИЦИЙ
}

(C) 2020 2. А.А. Жукова

\author{
ФИЦ «Информатика и управление» РАН (ФИЦ ИУ РАН) \\ НИУ «Высшая школа экономики» \\ МФТИ (ГУ) \\ sasha.mymail@gmail.com
}

Работа выполнена при поддержке гранта РФФИ, проект 17-01-00588 А «Динамические модели экономики со случайным шагом по времени».

DOI: $10.20948 / \mathrm{mm}-2020-04-02$

Работа рассматривает задачу фирмы, которая принимает решения относительно инвестиций, производства и выплаты дивидендов владельцам фирмы в условиях неопределенности моментов совершения транзакций. Ставится задача агента-производителя при конечном горизонте планирования. Используется подход к решению задач оптимального управления, возникающих в экономических моделях, с использованием метода множителей Лагранжа. Сформулированы достаточные условия оптимальности и выведена система уравнений в частных производных со сдвигом, определяющая решение задачи. Получено решение этой системы в случае постоянных цен и процентных ставок, а также в общем случае получены приближенные решения при высокой частоте транзакций. Показано отличие от задачи на бесконечном горизонте в силу наличия пограничного слоя, в котором анализ может значительно измениться по сравнению с анализом решения в пределах горизонта планирования. Данная модель имеет применение как блок агента-производителя в прикладном моделировании вычислимого межвременного равновесия экономики страны.

Ключевые слова: модель экономического агента, стохастическое оптимальное управление, метод множителей Лагранжа.

MODEL OF THE PRODUCER'S BEHAVIOR IN THE PRESENCE OF RANDOM MOMENTS OF OBTAINING A LOAN AND INVESTMENT

\section{A.A. Zhukova}

Federal Research Center "Computer Science and Control" of the RAS

Higher School of Economics - National Research University

MIPT

The presented work considers the problem of the firm, which makes decisions regarding investments, production and payment of dividends to the owners of the firm in the condi- 
tions of uncertainty in the timing of transactions. The task is set of the agent-producer on the finite planning horizon. An approach used to solve optimal control problem arising in the economic model is based on the method of Lagrange multipliers. Sufficient optimality conditions are formulated and a system of partial differential equations with a shift that determines the solution of the problem is derived. A solution to this system was obtained in the case of constant prices and interest rates, and in the general case, approximate solutions were obtained at a high transaction frequency. It shows the specifics of the problem compared to the problem on an infinite horizon due to the presence of a boundary layer in which the analysis can significantly change compared to the analysis of the solution within the planning horizon. This model might be used as a block of a manufacturing agent in applied modeling of the computable intertemporal equilibrium of a country's economy.

Keywords: model of an economic agent, stochastic optimal control, Lagrange multipliers method.

\section{1. Введение}

В данной статье представлен подход к решению задач оптимального управления, возникающих в экономических моделях, с использованием метода множителей Лагранжа. Этот метод используется в моделировании экономики не так широко, как мог бы, принимая во внимание его преимущества и удобство. Работы Грегори К. Чоу [1-3] продемонстрировали важность метода Лагранжа для экономического моделирования. Удобство этого метода для межвременного общего равновесия позволяет строить сложные структурные модели всей экономики и работать с системой дифференциальных и конечных уравнений вместо интегральных и функциональных, как в динамическом программировании [4,5]. Процедура формулировки условий оптимальности Лагранжа может быть в значительной степени преобразована в технологию получения набора отношений, готовых для дальнейшего аналитического или численного расчета [6,7] и проверок корректности [8].

Используя этот метод, можно решить не только детерминированные, но и стохастические экономические модели, хотя теория метода Лагранжа для стохастических моделей оптимального управления менее развита, чем метод динамического программирования [9]. Примеры полезности метода Лагранжа и попытки строгого вывода условий оптимальности для модели оптимального поведения экономического агента при наличии случайного процесса со скачками были приведены в статьях Ситу Ронга [10,11]. В этой статье мы развиваем их подход и уточняем конкретные математические конструкции, лежащие в основе математической постановки задачи стохастического управления.

Представленная статья демонстрирует метод Лагранжа на примере за- 
дачи фирмы, которая принимает решения относительно инвестиций, производства и выплаты дивидендов владельцам фирмы. Стохастическая составляющая в этой модели представлена случайным процессом моментов времени при совершении транзакций. Анализ основывается на математическом описании аналогичной модели оптимизации поведения потребителя [12]. Задача агента на конечном горизонте отличается от задачи на бесконечном горизонте наличием пограничного слоя, в котором решение может значительно измениться по сравнению с решением вдали от горизонта планирования [13].

Детали работы представлены в основном тексте статьи. Он организован следующим образом. В разделе 2 представлена формулировка модели. В разделе 3 сформулированы условия оптимальности в форме Лагранжа задачи стохастического оптимального управления. В разделе 4 представлен подход к решению условий оптимальности в форме синтеза (зависящего от состояния) управления. Раздел 5 делает заключительные замечания.

\section{2. Модель фирмы}

В предлагаемой модели поведения фирмы - производителя со случайной шкалой времени, фирма выступает инвестором, который

- в случайные моменты покупает инвестиционный продукт в размере $J(t)$ по неслучайной цене $p_{y}(t)$;

- $\quad$ в те же случайные моменты может брать и возвращать бессрочные кредиты в размере $K(t)$, в связи с чем непрерывно платит проценты по текущему долгу $L(t)$;

- $\quad$ может беспроцентно накапливать безналичные деньги, обозначаемые переменной $A(t)$;

- $\quad$ выпускает продукцию в объеме $Y(t)$ за счет использования производственных мощностей, описываемых переменной $M(t)$;

- $\quad$ продает продукцию на рынке по цене $p_{y}(t)$.

Пуассоновский поток случайных моментов смены состояния агента будем описывать процессом счета количества событий пуассоновского потока $\eta_{\omega}(t)$ на интервале времени $\left[t_{0}, t\right]$, где $\omega$ обозначает конкретную реализацию последовательности событий пуассоновского потока. Мы опустим индекс $\omega$ и будем обозначать записью $d \eta(t)$ изменение количества явлений пуассоновского потока в интервале времени $[t-d t, t]$ (оно может быть 0 или 1). Будем предполагать, что интенсивность пуассоновского процесса постоянна и равна $\Lambda$. В терминах приведенных переменных, деятельность фирмы формализуется следующим образом. 
Производство. Выпуск Y производится за счет эффективных фондов. Производственная функция, описывающая соотношение между размером производственных фондов и объемом выпуска, взята из широко используемой модели Харрода-Домара [14-16] $Y(t)=M(t) / b$.

Произведенный продукт реализуется по рыночной цене $p_{y}(t)$, которая предполагается внешней переменной для агента-производителя. Выручка от продажи произведенной продукции $p_{y}(t) Y(t)$. Для того, чтобы изменить размер эффективных фондов, производитель покупает инвестиционный продукт, за счет чего изменяются эффективные фонды: $d M(t)=J(t) d \eta(t)$, и затрачивает средства $p_{y}(t) J(t)$. Поток денег за период времени $[t-d t, t]$, связанный с производственной деятельностью, $p_{y}(t) Y(t) d t-p_{y}(t) J(t) d \eta(t)$.

Взаимодействие производителя с собственником. Производство принадлежит собственнику, который получает денежные дивиденды в размере $Z(t)$. Цель деятельности производителя - максимизация ожидаемой полезности по всем реализациям пуассоновского потока возможных сделок по кредитованию и инвестированию, извлекаемой владельцами фирмы от потока дивидендов

$$
\mathrm{E}\left\{\int_{t_{0}}^{T} U\left(Z(t) / p_{y}(t)\right) \exp (-\Delta t) d t\right\} \rightarrow \max .
$$

Здесь $U\left(Z(t) / p_{y}(t)\right)$ - моментальная полезность реальной (с поправкой на индекс цен $\left.p_{y}(t)\right)$ ценности дивидендов $Z(t)$. Предполагается, что $U$ строго возрастающая функция с $U^{\prime}(0)=\infty, U^{\prime}(\infty)=0$ и, в частности, что $U(X)=$ $=\ln (X)$ или $U(X)=\left(X^{a}-1\right) / a, a \in(0,1)$. Множитель $\exp (-\Delta t)$ моделирует степень «нетерпеливости» агента через параметр $\Delta$.

Финансовые операции. Банк предоставляет бессрочный кредит производителю под процент $r_{l}(t)$. В случайные моменты времени, описываемые пуассоновским потоком, долг производителя банку $L(t)$ меняется за счет выдачи кредита $d L(t)=K(t) d \eta(t)$. Поток денег за период времени $[t-d t, t]$, связанный с финансовыми операциями с банком, $K(t) d \eta(t)-r_{l}(t) L(t) d t$.

Все финансовые операции производителя суммируются в описании динамики его остатков денег на счетах, обозначенных переменной $A(t)$

$$
d A(t)=p_{y}(t) Y(t) d t-p_{y}(t) J(t) d \eta(t)+K(t) d \eta(t)-r_{l}(t) L(t) d t-Z(t) d t .
$$


Производитель не должен иметь возможность создавать так называемые «пузыри» - брать в долг неограниченные средства и платить за их счет дивиденды без попыток возврата долга. Это условие выполняется, если ввести следующие ограничения [17]: $A(t) \geq 0$ и терминальное ограничение $A(T)+p_{y}(T) M(T)-L(T) \geq 0$.

\section{3. Задача оптимального управления и условия оптимальности}

Приведенные выражения для различных аспектов деятельности производителя можно собрать вместе, чтобы записать формулировку задачи стохастического оптимального управления

$$
\begin{aligned}
& \mathrm{E}\left\{\int_{t_{0}}^{T} U\left(\frac{Z(t)}{p_{y}(t)}\right) e^{-\Delta t} d t\right\} \rightarrow \max , \\
& d A(t)=p_{y}(t) \frac{1}{b} M(t) d t-p_{y}(t) J(t) d \eta(t)+ \\
& \quad+K(t) d \eta(t)-r_{l}(t) L(t) d t-Z(t) d t, \\
& d L(t)=K(t) d \eta(t), \\
& d M(t)=J(t) d \eta(t), \\
& A(t) \geq 0, \\
& A(T)+p_{y}(T) M(T)-L(T) \geq 0 .
\end{aligned}
$$

Все процессы $A(\cdot), J(\cdot), K(\cdot), L(\cdot), M(\cdot), Z(\cdot)$ считаются непрерывными слева с определенным пределом справа процессами, адаптированными к естественной фильтрации $\left\{E_{t}\right\}_{t \geq 0}$, порожденной случайным пуассоновским процессом $\eta(\cdot)$. Все математические ожидания в данной статье понимаются как интегралы по мере на $\left\{E_{t}\right\}_{t \geq 0}$, определенной пуассоновским процессом $\eta(\cdot)$ с частотой $\Lambda$.

Задача состоит в поиске оптимального неупреждающего управления в виде трех процессов $J(\cdot), K(\cdot)$ и $Z(\cdot)$ такого, что при заданных начальных условиях $A\left(t_{0}\right), J\left(t_{0}\right), K\left(t_{0}\right), L\left(t_{0}\right), M\left(t_{0}\right), Z\left(t_{0}\right)$ процессы $A(\cdot), J(\cdot), K(\cdot), L(\cdot)$, $M(\cdot), Z(\cdot)$ удовлетворяют (4)-(6) и ограничениям (7), (8), а функционал (3) достигает максимума.

Решение задачи оптимального управления. Анализ этой задачи проведен по аналогии с задачей стохастического оптимального управления в 
модели поведения потребителя [10-13,18]. Кратко приведем принцип определения оптимального управления. Метод основывается на достаточных условиях оптимальности в форме Лагранжа. Функционал Лагранжа задачи (3)-(8) имеет вид

$$
\begin{aligned}
& \mathcal{L}_{\left[\tilde{\psi}_{1}(\cdot), \tilde{\psi}_{2}(\cdot), \tilde{\Psi}_{3}(\cdot), \tilde{\phi}_{4}(\cdot), \tilde{\Phi}_{1}\right]}[A(\cdot), L(\cdot), K(\cdot), M(\cdot), J(\cdot), Z(\cdot)]= \\
& =\mathrm{E}\left\{\int_{t_{0}}^{T} U\left(\frac{Z(t)}{p_{y}(t)}\right) \mathrm{e}^{-\Delta t} d t+\tilde{\psi}_{1}(t)(J(t) d \eta(t)-d M(t))+\right. \\
& +\tilde{\psi}_{2}(t)(K(t) d \eta(t)-d L(t))+ \\
& +\tilde{\psi}_{3}(t)\left(K(t) d \eta(t)-p_{y}(t) J(t) d \eta(t)-r_{l}(t) L(t) d t+\right. \\
& \left.\left.+\frac{p_{y}(t) M(t)}{b} d t-Z(t) d t-d A(t)\right)+\tilde{\phi}_{4}(t) A(t) d t\right\}+ \\
& +\tilde{\Phi}_{1}\left(A(T)+p_{y}(T) M(T)-L(T)\right) .
\end{aligned}
$$

Для этого функционала записываются условия оптимальности по аналогии с утверждениями 1-4 из работы [13], посвященной анализу модели потребителя. Для корректного определения функционала $\mathcal{L}$ определен класс, в котором ищем двойственные переменные. Считаем их достаточно гладкими функциями от $\{t, A(t+0), L(t+0), M(t+0)\}$. Поэтому полагаем, что

$$
\widetilde{\psi_{1,2,3}}(t)=\psi_{1,2,3}(t, A(t+0), L(t+0), M(t+0)) .
$$

Определенные так множители Лагранжа, оказываются непрерывными справа функциями времени с разрывами только в точках скачка пуассоновского процесса $\eta(t)$. Двойственная переменная к ограничению-неравенству $\tilde{\phi}_{4}(t)$, входит в условие дополняющей нежесткости, которое можно считать конечным уравнением, выражающим $\tilde{\phi}_{4}(t)$ через $A(t)$, что делает $\tilde{\phi}_{4}(t)$ непрерывной слева. Итак, все функции в функционале $\mathcal{L}$ считаются однозначными, кусочно-непрерывными и ограниченными. При таком построении интегралы по $d A(t), d L(t), d M(t)$ и $d \eta(t)$ понимаются в следующем смысле:

$$
\int_{a}^{b} \lambda(t) d n(t) \triangleq \sum_{k=0}^{\mathrm{T}} \lambda\left(\tau_{k}\right) \Delta n_{k}+\sum_{k=0}^{\mathrm{T}} \int_{\tau_{k}}^{\tau_{k+1}} \lambda(t) n^{\prime}(t) d t
$$

где $n, \lambda:[a, b] \rightarrow \mathbb{R}^{1}$ - кусочно-непрерывные функции, которые имеют на 
отрезке $[a, b]$ конечное число разрывов первого рода в точках $\tau_{k}, k=1, \ldots, T$, $\tau_{0} \triangleq a, \tau_{T+1} \triangleq b$, причем $n(\cdot)$ - дифференцируема между разрывами. Хотя формула интегрирования по частям неверна в произвольном случае, в рассматриваемом случае она имеет место.

Достаточные условия оптимальности. Можно доказать утверждения, аналогичные утверждениям для модели поведения потребителя [13]. Первые два утверждения приведем из статьи [13], вторые два утверждения являются новыми результатами для предлагаемой новой стохастической модели поведения потребителя.

Утвержндение 1. Если $n(\cdot)$ непрерывна слева, а $\lambda(\cdot)$ непрерывна справа, а между разрывами обе функции гладкие, и $a, b$ не являются точками их разрыва, то для (11) выполняется соотношение

$$
\int_{a}^{b} \lambda(t) d n(t)=n(b) \lambda(b)-n(a) \lambda(a)-\int_{a}^{b} n(t) d \lambda(t) .
$$

Утвержндение 2. Пусть $n, \lambda$ - измеримые относительно меры Н процессы (неважно неупреждающче или нет) с кусочно-непрерывными (неважно справа или слева) экспоненциально ограниченныли реализациями, причем реализачии $\lambda$ могут иметь разрывы только в моментьл $\tau_{k}$ скачков проиесса $\eta$, а в промежутках $\lambda$ имеет производную $d \lambda / d t$. Тогда

$$
\mathbf{E}\left\{\int_{a}^{\infty} n(t) d \lambda(t)\right\}=\int_{a}^{\infty} \mathbf{E}\left\{n(t) \frac{d \lambda}{d t}(t)+\Lambda \mathbf{E}\left\{n(t) \Delta \lambda(t) \mid t=\tau_{k}\right\}\right\} d t
$$

где $\mathbf{E}\left\{\cdot \mid t=\tau_{k}\right\}$ ожидание при условии, что в момент $t$ происходит событие пуассоновского потока $\eta$. Оно определено корректно, поскольку $\tau_{k}-$ марковский момент.

Утверждения 1 и 2 используются для формулировки утверждений относительно седловой точки функционала Лагранжа.

Утверждение 3. Пусть для некоторых непрерывных справа (не обязательно неупреждающих) прочессов $\tilde{\psi}_{1}(t), \tilde{\psi}_{2}(t), \tilde{\psi}_{3}(t) \tilde{\phi}_{4}(t) \geq 0, u$ случайной величины $\Phi_{1} \geq 0$ таких, что интеграл в (9) с учетом определения (11) сходится при всех

$$
\langle A(\cdot), L(\cdot), M(\cdot), K(\cdot), J(\cdot), Z(\cdot)\rangle \in \mathbb{L} \mathbb{C} \times \mathbb{L} \mathbb{C} \times \mathbb{L} \mathbb{C} \times \mathbb{L} \mathbb{C} \times \mathbb{L} \mathbb{C} \times \mathbb{L} \mathbb{C}^{1},
$$

найдется тройка неупреждающих процессов

${ }^{1}$ Под $\mathbb{L} \mathbb{C}$ понимается пространство непрерывных слева ограниченных функций. 
Модель поведения производителя при наличии случайных моментов ...

$$
\left\langle\hat{A}_{\eta}(\cdot), \hat{L}_{\eta}(\cdot), \hat{M}_{\eta}(\cdot), \hat{K}_{\eta}(\cdot), \hat{J}_{\eta}(\cdot), \hat{Z}_{\eta}(\cdot)\right\rangle \in \mathbb{L} \mathbb{C} \times \mathbb{L} \mathbb{C} \times \mathbb{L} \mathbb{C} \times \mathbb{L} \mathbb{C} \times \mathbb{L} \mathbb{C} \times \mathbb{L} \mathbb{C},
$$

удовлетворяющих заданнымм начальнымм условиям на $A(0), L(0), M(0)$, коmopble

а) доставляют максимум функционалу Лагранжа (9)

$$
\begin{aligned}
& \left\langle\hat{A}_{\eta}(\cdot), \hat{L}_{\eta}(\cdot), \hat{M}_{\eta}(\cdot), \hat{K}_{\eta}(\cdot), \hat{J}_{\eta}(\cdot), \hat{Z}_{\eta}(\cdot)\right\rangle \in \\
& \in \underset{\langle A(\cdot), L(\cdot), M(\cdot), K(\cdot), J(\cdot), Z(\cdot)\rangle}{\operatorname{Argmax}} \mathcal{L}_{\left[\tilde{\psi}_{1}(\cdot), \tilde{\Psi}_{2}(\cdot), \tilde{\Psi}_{3}(\cdot), \tilde{\phi}_{4}(\cdot), \Phi_{1}\right]}[A(\cdot), L(\cdot), M(\cdot), K(\cdot), J(\cdot), Z(\cdot)],
\end{aligned}
$$

по множеству неупреждающих прощессов $\langle A(\cdot), L(\cdot), M(\cdot), K(\cdot), J(\cdot), Z(\cdot)\rangle$ c реализациями из $\mathbb{L} \mathbb{C} \times \mathbb{L} \mathbb{C} \times \mathbb{L} \mathbb{C} \times \mathbb{L} \mathbb{C} \times \mathbb{L} \mathbb{C} \times \mathbb{L} \mathbb{C}$ с заданныли начальными условиями $A(0), L(0), M(0)$.

b) почти наверное при всех $t$ удовлетворяют условиям дополняющей нежесткости

$$
\begin{aligned}
& d \hat{L}_{\eta}(t)=\hat{K}_{\eta}(t) d \eta(t), \quad d \hat{M}_{\eta}(t) d t=\hat{J}_{\eta}(t) d \eta(t) \\
& d \hat{A}_{\eta}(t)=\hat{K}_{\eta}(t) d \eta(t)-r_{l}(t) \hat{L}_{\eta}(t) d t+\frac{p_{y}(t)}{b} \hat{M}_{\eta}(t) d t-p_{y}(t) \hat{J}_{\eta}(t) d \eta(t)-Z(t) d t \\
& \hat{A}_{\eta}(t) \geq 0, \hat{A}_{\eta}(T)+p_{y}(T) \hat{M}_{\eta}(T)-\hat{L}_{\eta}(T) \geq 0, \quad \tilde{\phi}_{4}(t) \geq 0, \quad \tilde{\Phi}_{1}(t) \geq 0 \\
& \tilde{\phi}_{4}(t) \hat{A}_{\eta}(t)=0, \quad \tilde{\Phi}_{1}\left(\hat{A}_{\eta}(T)+p_{y}(T) \hat{M}_{\eta}(T)-\hat{L}_{\eta}(T)\right)=0 .
\end{aligned}
$$

Тогда $\left\langle\hat{A}_{\eta}(\cdot), \hat{L}_{\eta}(\cdot), \hat{M}_{\eta}(\cdot), \hat{K}_{\eta}(\cdot), \hat{J}_{\eta}(\cdot), \hat{Z}_{\eta}(\cdot)\right\rangle$-решение задачи (3)-(8).

При поиске максимума функционала Лагранжа интегрируются по частям слагаемые, содержащие дифференциалы фазовых переменных $(d A(t)$, $d L(t), d M(t))$. После этого функционал Лагранжа варьируется по фазовым переменным $A(\cdot), L(\cdot), M(\cdot)$ и управлениям $K(\cdot), J(\cdot), Z(\cdot)$, чтобы получить достаточные условия оптимальности.

Утвержндение 4. Если с вероятностью 1 процессы $\tilde{\psi}_{1}(t), \tilde{\psi}_{2}(t), \tilde{\psi}_{3}(t)$, $\tilde{\phi}_{4}(t) \geq 0$ гладкие между скачками процесса $\eta$, то для выполнения условия (a) Утверждения 3 достаточно выполнения с вероятностью 1 следующих соотношений

$$
\begin{aligned}
& \mathbf{E}_{\eta[t, T)}\left\{\Lambda \mathbf{E}\left\{\tilde{\psi}_{3}(t)-\tilde{\psi}_{3}(t-0) \mid t=\tau_{k}\right\}+\frac{d}{d t} \tilde{\psi}_{3}(t)+\tilde{\phi}_{4}(t) d t\right\}=0, \\
& \mathbf{E}_{\eta[t, T)}\left\{\mathbf{E}\left\{\tilde{\psi}_{1}(t) \mid t=\tau_{k}\right\}+p_{y}(t) \mathbf{E}\left\{\tilde{\psi}_{3}(t) \mid t=\tau_{k}\right\}\right\}=0,
\end{aligned}
$$




$$
\begin{aligned}
& \mathbf{E}_{\eta[t, T)}\left\{\mathbf{E}\left\{\tilde{\Psi}_{2}(t) \mid t=\tau_{k}\right\}+\mathbf{E}\left\{\tilde{\Psi}_{3}(t) \mid t=\tau_{k}\right\}\right\}=0, \\
& \mathbf{E}_{\eta[t, T)}\left\{\Lambda \mathbf{E}\left\{\tilde{\Psi}_{2}(t)-\tilde{\psi}_{2}(t-0) \mid t=\tau_{k}\right\}+\frac{d}{d t} \tilde{\psi}_{2}(t)-r_{l}(t) \tilde{\Psi}_{3}(t) d t\right\}=0, \\
& \mathbf{E}_{\eta[t, T)}\left\{\Lambda \mathbf{E}\left\{\tilde{\Psi}_{1}(t)-\tilde{\psi}_{1}(t-0) \mid t=\tau_{k}\right\}+\frac{d}{d t} \tilde{\psi}_{1}(t)+\frac{p_{y}(t)}{b} \psi_{3}(t) d t\right\}=0, \\
& \mathbf{E}_{\eta[t, T)}\left\{D(U)\left(\hat{Z}_{\eta}(t) / p_{y}(t)\right) \exp (-\Delta t)-p_{y}(t) \tilde{\Psi}_{3}(t)\right\}=0, \\
& \mathbf{E}_{\eta[T-d t, T)}\left\{-\tilde{\Psi}_{1}(T)+p_{y}(T) \tilde{\Phi}_{1}\right\}=0, \quad \mathbf{E}_{\eta[T-d t, T)}\left\{\tilde{\psi}_{2}(T)+\tilde{\Phi}_{1}\right\}=0, \\
& \mathbf{E}_{\eta[T-d t, T)}\left\{\tilde{\Psi}_{3}(T)-\tilde{\Phi}_{1}\right\}=0 .
\end{aligned}
$$

Здесь $E_{\eta[a, b)}$ - условное математическое ожидание при заданном $\rceil[0, a)$. Дальнейший анализ задачи оптимального стохастического управления заключается в исследовании системы уравнений Утверждения 4, в котором будут рассматриваться только оптимальные процессы

$$
\left\langle\hat{A}_{\eta}(\cdot), \hat{L}_{\eta}(\cdot), \hat{M}_{\eta}(\cdot), \hat{K}_{\eta}(\cdot), \hat{J}_{\eta}(\cdot), \hat{Z}_{\eta}(\cdot)\right\rangle,
$$

поэтому индекс $\eta$ и «шляпку» в их обозначении опускаем. В соотношениях Утверждения 4 остается учесть (10)

$$
\begin{aligned}
& \mathbf{E}\left\{\tilde{\psi}_{1,2,3}(t) \mid t=\tau_{k}\right\}=\psi_{1,2,3}\left(t, A(t)-p_{y}(t) J(t)+K(t), L(t)+K(t), M(t)+J(t)\right), \\
& \mathbf{E}\left\{\left(\tilde{\psi}_{1,2,3}(t)-\tilde{\psi}_{1,2,3}(t-0)\right) \mid t=\tau_{k}\right\}= \\
&=\psi_{1,2,3}\left(t, A(t)-p_{y}(t) J(t)+K(t), L(t)+K(t), M(t)+J(t)\right)-\psi_{1,2,3}(t, A(t), L(t), M(t)) .
\end{aligned}
$$

Между событиями пуассоновского потока смены состояния агента, согласно (4)-(6) $d L(t)=0, d M(t)=0$,

$$
d A(t)=p_{y}(t) \frac{1}{b} M(t) d t-r_{l}(t) L(t) d t-Z(t) d t,
$$

поэтому

$$
\begin{aligned}
& \frac{d}{d t} \psi_{1,2,3}(t)=\frac{\partial}{\partial t} \psi_{1,2,3}(t, A(t), L(t), M(t))+ \\
& +\left(p_{y}(t) \frac{1}{b} M(t) d t-r_{l}(t) L(t) d t-Z(t) d t\right) \frac{\partial}{\partial A} \psi_{1,2,3}(t, A(t), L(t), M(t)) .
\end{aligned}
$$




\section{4. Решение задачи фирмы}

Как отмечалось выше, естественно искать $\tilde{\phi}_{4}(t)$ как неупреждающий непрерывный слева процесс. Поскольку реализации процесса $\langle A(t), L(t), M(t)\rangle$ блуждают по $\mathbb{R}_{+} \times \mathbb{R} \times \mathbb{R}$, выполнения достаточных условий оптимальности, как и в модели поведения потребителя, естественно требовать не вдоль отдельной траектории, а тождественно по всему пространству состояний. Однако, в отличие от модели поведения потребителя, пространство $\langle A(t), L(t), M(t)\rangle$ на одно измерение больше. Тогда, считая $J, M$ и $K$ функциями состояния,

$$
\begin{aligned}
& J(t)=J(t, A(t), L(t), M(t)), \quad M(t)=M(t, A(t), L(t), M(t)), \\
& K(t)=K(t, A(t), L(t), M(t)),
\end{aligned}
$$

получаем уравнения для определения двойственных переменных.

$$
\begin{aligned}
& \phi_{4}(t, A, L, M) A=0, \\
& -\phi_{4}(t, A, L, M)-\frac{\partial}{\partial t} \psi_{3}(t, A, L, M)+r_{l}(t) L \frac{\partial}{\partial A} \psi_{3}(t, A, L, M)- \\
& -\frac{p_{y}(t) M}{b} \frac{\partial}{\partial A} \psi_{3}(t, A, L, M)+\frac{\partial}{\partial A} \psi_{3}(t, A, L, M) Z(t, A, L, M)+ \\
& +\Lambda \psi_{3}(t, A, L, M)-\Lambda \psi_{3}(t, K(t, A, L, M)+A- \\
& \left.-p_{y}(t) J(t, A, L, M), K(t, A, L, M)+L, J(t, A, L, M)+M\right)=0, \\
& p_{y}(t) \psi_{3}\left(t, K(t, A, L, M)+A-p_{y}(t) J(t, A, L, M), K(t, A, L, M)+\right. \\
& +L, J(t, A, L, M)+M)=\psi_{1}(t, K(t, A, L, M)+A- \\
& \left.-p_{y}(t) J(t, A, L, M), K(t, A, L, M)+L, J(t, A, L, M)+M\right), \\
& \psi_{2}\left(t, K(t, A, L, M)+A-p_{y}(t) J(t, A, L, M), K(t, A, L, M)+\right. \\
& +L, J(t, A, L, M)+M)=-\psi_{3}(t, K(t, A, L, M)+A- \\
& \left.-p_{y}(t) J(t, A, L, M), K(t, A, L, M)+L, J(t, A, L, M)+M\right), \\
& r_{l}(t) \psi_{3}(t, A, L, M)-\frac{\partial}{\partial t} \psi_{2}(t, A, L, M)+r_{l}(t) L \frac{\partial}{\partial A} \psi_{2}(t, A, L, M)- \\
& -\frac{p_{y}(t) M}{b} \frac{\partial}{\partial A} \psi_{2}(t, A, L, M)+Z(t, A, L, M) \frac{\partial}{\partial A} \psi_{2}(t, A, L, M)- \\
& -\Lambda \psi_{3}(t, A, L, M)-\Lambda \psi_{2}\left(t,-p_{y}(t) J(t, A, L, M)+K(t, A, L, M)+\right. \\
& +A, K(t, A, L, M)+L, J(t, A, L, M)+M)=0,
\end{aligned}
$$




$$
\begin{aligned}
& -\frac{\partial}{\partial t} \psi_{1}(t, A, L, M)+r_{l}(t) L \frac{\partial}{\partial A} \psi_{1}(t, A, L, M)+Z(t, A, L, M) \frac{\partial}{\partial A} \psi_{1}(t, A, L, M)+ \\
& +\Lambda \psi_{3}(t, A, L, M) p_{y}(t)-\Lambda \psi_{3}(t, A, L, M) \frac{d}{d t} p_{y}(t)- \\
& -\frac{p_{y}(t)}{b}\left(\psi_{3}(t, A, L, M)+M \frac{\partial}{\partial A} \psi_{1}(t, A, L, M)\right)- \\
& -\Lambda \psi_{1}\left(t,-p_{y}(t) J(t, A, L, M)+K(t, A, L, M)+\right. \\
& +A, K(t, A, L, M)+L, J(t, A, L, M)+M)=0 \\
& \quad U^{\prime}\left(Z(t, A, L, M) / p_{y}(t)\right)=\psi_{3}(t, A, L, M) p_{y}(t) \exp (\Delta t) \\
& \psi_{1}(T, A, L, M)=p_{y}(T) \Phi_{1}, \quad \psi_{2}(T, A, L, M)=-\Phi_{1}, \quad \psi_{3}(T, A, L, M)=\Phi_{1}, \\
& \quad \Phi_{1}\left(A(T)+p_{y}(T) M(T)-L(T)\right)=0 .
\end{aligned}
$$

4.1. Анализ достаточных условий оптимальности. Мы ищем асимптотическое решение, в котором частота транзакций $\Lambda$ велика. Поэтому модель приближается к модели непрерывных возможностей совершать транзакции - детерминированной модели. Мы предполагаем, что функция полезности логарифмическая. Детерминированная модель известна в макроэкономике и имеет решение, при котором денежные остатки равны нулю [4], и имеют место следующие отношения:

$$
\begin{aligned}
& M(t) p_{y}(t)-L(t)=\exp \left(-\int_{t}^{T} r_{l}(u) d u\right) \int_{t}^{T} Z(\tau) \exp \left(\int_{\tau}^{T} r_{l}(u) d u\right) d \tau \\
& 0=-r_{l}(t)+b^{-1}+\frac{d p_{y}(t) / d t}{p_{y}(t)}
\end{aligned}
$$

При анализе асимптотической версии модели со случайными скачками мы также пытаемся найти решение с нулевым денежным балансом, предполагая аналогию с детерминированной версией. Это усложняется тем, что на интервале времени выделяются две области $[0, T]$. Первая - при $t$ много меньших $T$, что позволяет применить асимптотическое разложение Лапласа интегральных выражений. Вторая - при $\Lambda(T-t)$ порядка $O(1)$, что позволяет исследовать уравнения модели вблизи горизонта планирования. Основные этапы анализа и приближенные решения представлены ниже.

Режим при $A(t)=0$ из-за скачков переменных добавляет два условия 
Модель поведения производителя при наличии случайных моментов ...

$$
\begin{aligned}
& A(t-0)=-r_{l}(t) L+\frac{1}{b} M p_{y}(t)-Z(t, A, L, M) b=0, \\
& A(t+0)-A(t-0)=K(t, A, L, M)-p_{y}(t) J(t, A, L, M)=0 .
\end{aligned}
$$

Так как переменная $A(t)$ равна нулю, мы опускаем ее как аргумент управления и двойственных переменных. Следующие замены упрощают анализ:

$$
\begin{aligned}
& K(t, A, L, M)=k(t, L, M), \quad J(t, A, L, M)=j(t, L, M), \\
& \Psi_{1}(t, A, L, M)=-\exp (\Lambda t) p_{y}(t) \Psi_{2}(t, L, M)+\exp (\Lambda t) \Xi(t, L, M), \\
& \Psi_{2}(t, A, L, M)=\exp (\Lambda t) \Psi_{2}(t, L, M), \quad \Psi_{3}(t, A, L, M)=\Psi_{3}(t, L, M) .
\end{aligned}
$$

Дифференциальные и функциональные уравнения (12)-(20) сводятся к

$$
\begin{aligned}
& \Lambda \int_{t}^{T} \frac{\exp (-\tau \Lambda-\tau \Delta)}{F(\tau, t, L, M)} d \tau+ \\
& +p_{y}(T) \Phi_{1}\left(p_{y}(T)(j(t, L, M)+M)-p_{y}(t) j(t, L, M)-L\right) \exp (-\Lambda T)- \\
& -\int_{t}^{T} \frac{p_{y}(\tau) \exp (-\tau \Lambda-\tau \Delta)}{r_{l}(\tau) b p_{y}(t) j(t, L, M)+r_{l}(\tau) L b-p_{y}(\tau) j(t, L, M)-M p_{y}(\tau)} d \tau+ \\
& +\frac{b \exp (-\Lambda t-\Delta t) p_{y}(t)}{r_{l}(t) b p_{y}(t) j(t, L, M)+r_{l}(t) L b-p_{y}(t) j(t, L, M)-M p_{y}(t)}=0,
\end{aligned}
$$

где

$$
\begin{aligned}
& F(\tau, t, L, M)=-\frac{r_{l}(\tau) L b-M p_{y}(\tau)}{p_{y}(\tau) b}-\frac{\left(-p_{y}(\tau)+r_{l}(\tau) b p_{y}(t)\right) j(t, L, M)}{p_{y}(\tau) b}- \\
& -\frac{\left(-1+r_{l}(\tau) b\right) j\left(\tau, p_{y}(t) j(t, L, M)+L, j(t, L, M)+M\right)}{b} .
\end{aligned}
$$

4.2. Случай постоянных цен и процентной ставки. Случай $r_{l}(t)=r$, $p_{y}(t)=p$ может служить иллюстрацией. В этом случае уравнение (24) упрощается заменой

$$
r b p j(t, L, M)+r L b-p j(t, L, M)-M p=(G(t, L, M))^{-1} .
$$

Следовательно, функциональное уравнение принимает вид

$$
\begin{aligned}
& \frac{p G(t, L, M)(-1+\Lambda b+b \Delta) \mathrm{e}^{-\Lambda t-\Delta t}}{\Lambda+\Delta}+\frac{p G(t, L, M) \mathrm{e}^{-\Lambda T-T \Delta}}{\Lambda+\Delta}-p b \Lambda \times \\
& \times \int_{t}^{T} \mathrm{e}^{-\tau(\Lambda+\Delta)} G\left(\tau, \frac{1+(M p-L) G(t, L, M)}{G(t, L, M)(r b-1)}, \frac{1-(r L b-M p) G(t, L, M) r b}{p G(t, L, M)(r b-1)}\right) d \tau=0 .
\end{aligned}
$$


Видно, что переменные $L, M$ не входят в это уравнение нигде, кроме аргументов неизвестной функции. Это позволяет предположить, что решение имеет вид $G(t, L, M)=g(t, M p-L)$. Тогда можно получить решение

$$
\begin{aligned}
& j(t, L, M)=-\frac{r L b-M p}{p(r b-1)}+ \\
& +\frac{((b \Lambda+b \Delta-1) \exp (-\Lambda t-\Delta t)+\exp (-T(\Lambda+\Delta)))^{(b \Delta-1) /(\Lambda b+b \Delta-1)}}{f_{0}(M p-L) p(r b-1)} .
\end{aligned}
$$

Функция $f_{0}(M p-L)$ является константой в силу (22), т.к. комбинация $M(t) p-L(t)$ оказывается постоянной во времени. Двойственная к $A$ в условиях оптимальности (12), действительно, ненулевая, т.к. ее выражение

$$
\phi_{4}(t, A, L, M)=-\frac{(\Lambda+\Delta) b \exp (-\Delta t)}{r L b-M p}+\Lambda b f_{0}(M p-L) \exp (-(t / b)),
$$

что подтверждает предположение, которое мы сделали вначале о режиме нулевых денежных остатков.

Случай непостоянной цены и процентной ставки является более сложным, поскольку он не позволяет нам найти точное решение для (24), но может быть исследован асимптотическими методами. При $t \ll T$ применяется асимптотическое приближение интеграла типа Лапласа в (24) при большом $\Lambda$, что дает приближенную версию уравнения (24)

$$
j\left(t, p_{y}(t) j(t, L, M)+L, j(t, L, M)+M\right)=\frac{r_{l}(t) L b-M p_{y}(t)}{p_{y}(t)(\Lambda b-1)\left(r_{l}(t) b-1\right)}+\frac{j(t, L, M)}{\Lambda b-1} .
$$

Оно имеет линейное решение

$$
j(t, L, M)=\frac{r_{l}(t) L b}{p_{y}(t)(\Lambda b-1)\left(r_{l}(t) b-1\right)}-\frac{M}{(\Lambda b-1)\left(r_{l}(t) b-1\right)} .
$$

При $t \sim T$ появляется пограничный слой, где асимптотическое разложение интеграла больше не применимо. Тогда заменой $t=T-(\theta / \Lambda)$ и разложением в ряд по $\Lambda$ выражений в (24) получаем асимптотическое выражение управления

$$
\begin{aligned}
& j(t, L, M)=-\frac{r_{l}(T) L b-p_{y}(T) M}{p_{y}(T)\left(r_{l}(T) b-1\right)}- \\
& -\frac{\exp (-\Delta T) b}{\Phi_{1}\left(p_{y}(T) M-L\right)\left(r_{l}(T) b-1\right) p_{y}(T)}+O(1 / \Lambda) .
\end{aligned}
$$


Выражение порядка $O(1 / \Lambda)$ получено, но не приведено в силу сложности. Важный вопрос заключается в том, согласуется ли предположение $A(t)=0$ с системой условий оптимальности. Другими словами, найденное решение (32) должно соответствовать двойственными переменным, согласующимся с нашим предположением $A(t)=0$. Можно показать, что в обоих случаях это так.

\section{5. Заключение}

В данной работе предложена модель поведения производителя-инвестора при наличии случайности во времени кредитования и инвестирования. Таким образом, предполагается моделировать неравномерную сетку времени в описании экономического агента. Предложен метод анализа модели и построено оптимальное поведение производителя. Обнаружен эффект связи между процентными ставками и ценами, аналогичный тому, который наблюдается в детерминированной модели. Следствием такого эффекта является ограничение на действия экономического агента, который может координировать финансовые потоки в равновесии такой системы - банка или финансового рынка. Данное исследование может быть использовано для построения блока агента-производителя и агента-банка в модели общего равновесия со случайной временной сеткой.

\section{СПИСОК ЛИТЕРАТУРЫ}

1. G.C. Chow. The Lagrange method of optimization with applications to portfolio and investment decisions // J. of Economic Dynamics and Control, North-Holland, 1996, v.20, №1-3, p.1-18.

2. G.C. Chow. Dynamic Economics: Optimization by the Lagrange Method. - Oxford: Oxford University Press, 1997, $248 \mathrm{p}$.

3. G.C. Chow. Optimal control without solving the Bellman equation // Journal of Economic Dynamics and Control, North-Holland, 1993, v.17, № 4, p.621-630.

4. М.Ю. Андреев, В.П. Вржещ, Н.П. Пильник, И.Г. Поспелов, М.А. Хохлов, А.А. Жукова, C.A. Радионов. Модель межвременного равновесия экономики России, основанная на дезагрегировании макроэкономического баланса // Тр. сем. им. И.Г. Петровского. - М.: Изд-во Моск. ун-та, 2013, т.29, с.43-145; англ. пер. M.Y. Andreyev, V.P. Vrzheshch, N.P. Pilnik, I.G. Pospelov, M.A. Khokhlov, A.A. Zhukova, S.A. Radionov. Intertemporal general equilibrium model of the Russian economy based on national accounts deaggregation // J. of Math. Scienc., 2014, v.197, № 2, p.175-236.

5. N.P. Pilnik, I.G. Pospelov, S.A. Radionov, A.A. Zhukova. The intertemporal general equilibrium model of the economy with the product, money and stock markets // International Journal of Computational Economics and Econometrics, 2014, v.4, № 1/2, p.207.

6. M.A. Khokhlov, I.G. Pospelov, L.Y. Pospelova. Technology of development and implemen- 
tation of realistic (country-specific) models of intertemporal equilibrium // International Journal of Computational Economics and Econometrics, 2014, v 4, № 1/2, p.234.

7. А.А. Петров, И.Г. Поспелов. Математические модели экономики России // Вестник PAH, 2009, т.79, № 6, c.492-506.

англ. пер. A.A. Petrov, I.G. Pospelov. Mathematical models of the Russian economy // Her. Russ. Acad. Sci., SP MAIK Nauka/Interperiodica, 2009, v. 79, № 3, p.205-216.

8. И.Г. Поспелов, М.А. Хохлов. Метод проверки размерности для исследования моделей экономической динамики // Математ. моделирование, 2006, v.18, №10, p.113-122.

I.G. Pospelov, M.A. Khokhlov. Metod proverki razmernosti dlia issledovaniia modelei ekonomicheskoi dinamiki // Matematicheskoe modelirovanie, 2006, v.18, №10, p.113-122.

9. B.K. Øksendal, A. Sulem. Applied stochastic control of jump diffusions. - Berlin: Springer, 2007, $257 \mathrm{p}$.

10. S. Rong. Optimization for a financial market with jumps by Lagrange's method // Pacific Econ. Rev., John Wiley \& Sons, 1999, v.4, № 3, p.261-276.

11. S. Rong. Theory of stochastic differential equations with jumps and applications: mathematical and analytical techniques with applications to engineering. - Berlin: Springer, 2006, $434 \mathrm{p}$.

12. А.А. Жукова, И.Г. Поспелов. Стохастическая модель торговли неликвидным товаром // Труды МФТИ, 2012, т.4, №2, с.131-147.

I.G. Pospelov, A.A. Zhukova. Stokhasticheskaya model' torgovli nelikvidnym tovarom // Trudy MFTI, 2012, t.4, № 2, s.131-147.

13. А.А. Жукова, И.Г. Поспелов. Модель оптимального потребления при наличии возможности кредитования в случайные моменты времени // Экономический журнал ВШЭ, 2018, т.22, № 3, с.330-361.

I.G. Pospelov, A.A. Zhukova. Model' optimal'nogo potrebleniya pri nalichii vozmozhnosti kreditovaniya v sluchaynyye momenty vremeni // Ekonomicheskiy Zhurnal VSHE, 2018, v.22, № 3. s.330-361.

14. R.F. Harrod. An Essay in Dynamic Theory // Econ. J. 1939, v.49, № 193, p.14-33.

15. E.D. Domar. Capital Expansion, Rate of Growth, and Employment // Econometrica, 1946, v.14, № 2, p.137.

16. D. Acemoglu. Introduction to modern economic growth. - NJ.: Princeton University Press, 2009, $990 \mathrm{p}$.

17. И.Г. Поспелов, Н.П. Пильник. О естественных терминальных условиях в моделях межвременного равновесия // Экономический журнал ВШЭ, 2007, т.11, № 1, с.1-33.

I.G. Pospelov, N.P. Pil'nik. O yestestvennykh terminal'nykh usloviyakh v modelyakh mezhvremennogo ravnovesiya // Ekonomicheskiy zhurnal VSHE, 2007, t.11, № 1, s.1-33.

18. А.А. Жукова, И.Г. Поспелов. Исследование стохастической модели сбережений с инерционностью потребления // Труды МФТИ, 2014, т.6, № 4, с.41-48;

I.G. Pospelov, A.A.Zhukova. Issledovaniye stokhasticheskoy modeli sberezheniy s inertsionnost'yu potrebleniya // Trudy MFTI, 2014, t.6, № 4, s.41-48.

Поступила в редакцию 21.10.2019

После доработки 21.10.2019

Принята к публикации 25.11.2019 